\title{
Glosario de Medicina Basada en Evidencia
}

\author{
Ignacio Neumann $\mathbf{B}^{1}$.
}

\begin{abstract}
Allocation concealment (ocultamiento de la secuencia de asignación): Forma de asignación a los grupos intervenidos y control cuya secuencia se mantiene oculta.

Aplicabilidad (validez externa): Grado en el que los resultados de un estudio son aplicables a distintos escenarios clínicos.

Estudio ciego: Estudio en el cual todos los participantes (sujetos en estudio, tratantes, recolectores de datos, adjudicadores de la ocurrencia del evento y analistas de los datos) desconocen la distribución de los pacientes en los distintos grupos. En la actualidad, los términos simple, doble o triple ciego tienden a ser reemplazados por la descripción de cuáles participantes fueron ciegos en cada estudio.
\end{abstract}

Estudio randomizado (estudio aleatorio): Estudio en el cual la asignación a los grupos en comparación se efectúa al azar. En consecuencia, cada sujeto tiene igual probabilidad de ingresar a cada grupo.

Intención de tratar: Método por el cual todos los sujetos en estudio son analizados dentro del grupo al que originalmente fueron asignados, independiente de si recibieron 0 no la intervención.

Intervalo de confianza: Es el intervalo dentro del cual se estima, con cierto grado de certeza predeterminada (habitualmente 95\%), que se encuentra el valor real de una variable para una población.

Likelihood ratio (cociente de probabilidad): Razón entre la probabilidad de que un examen tenga cierto resultado en los pacientes que tienen la enfermedad versus los que no la tienen. Es la propiedad del examen de modificar la probabilidad de un diagnóstico específico después de aplicarlo.

Metanálisis: Utilización de herramientas estadísticas para integrar los resultados de los estudios incluidos en una revisión sistemática.

${ }^{1}$ Interno de Medicina, PUC

Revisado por la Unidad de Medicina Basada en Evidencia UC (UMBE-UC) 
Número necesario para hacer daño: Es el número de pacientes que habría que tratar para que aparezca un efecto adverso del tratamiento en un paciente adicional.

Número necesario para tratar: Es el número de pacientes que habría que tratar para que en un paciente adicional se obtenga un desenlace favorable o se prevenga un desenlace desfavorable.

Odds (chance): Cociente entre la ocurrencia y la no ocurrencia de un evento.

Odds ratio (razón de chance): Cociente entre el odds del evento en el grupo intervenido y el odds del evento en el grupo control.

Outcome (desenlace o resultado): Evento observado en el estudio.

Reducción de riesgo absoluto o diferencia de riesgo: Diferencia en la tasa de eventos entre el grupo control y el grupo intervenido.

Reducción de riesgo relativo: Proporción en que se disminuye el riesgo en el grupo intervenido respecto del grupo control.

Revisión sistemática: Revisión de un tema clínico específico utilizando métodos explícitos para identificar, seleccionar y evaluar críticamente los estudios relevantes. Se pueden utilizar o no métodos estadísticos (metanálisis) para analizar y resumir los resultados de los estudios incluidos.

Riesgo relativo: Cociente entre la tasa de eventos del grupo intervenido y el grupo control. Es el riesgo residual del grupo que recibió la intervención.

Sesgo: Error sistemático.

Validez (validez interna): Grado en el cual el resultado de un estudio se acerca a la realidad mediante una metodología que minimiza el sesgo.

\section{FóRMULAS ÚtIILS EN MBE}

Outcome

\begin{tabular}{l|c|c|}
\multicolumn{2}{c}{} & \multicolumn{2}{c}{ Outcome } \\
Presente & Ausente \\
\cline { 2 - 3 } Grupo intervenido & $\mathrm{a}$ & $\mathrm{b}$ \\
\cline { 2 - 3 } Grupo control & $\mathrm{c}$ & $\mathrm{d}$ \\
\hline
\end{tabular}

Reducción de riesgo absoluto (RRA): $(\mathrm{c} / \mathrm{c}+\mathrm{d})-(\mathrm{a} / \mathrm{a}+\mathrm{b})$

Riesgo relativo (RR): $(\mathrm{a} / \mathrm{a}+\mathrm{b}) /(\mathrm{c} / \mathrm{c}+\mathrm{d})$

Reducción de riesgo relativo (RRR): 1-RR

Odds ratio (OR): (a/b) / (c/d)

Número necesario para tratar (NNT): (1/RRA) x 100 